\title{
Processing Ultrafast Optical Signals in Broadband Telecom Systems by means of Cascaded Quadratic Nonlinearities
}

\author{
Katia Gallo, Jerry Prawiharjo, Francesca Parmigiani, Paulo Almeida, \\ Periklis Petropoulos and David J. Richardson \\ Optoelectronics Research Centre,University of Southampton, SO17 1 BJ Southampton, UK \\ Tel: (44)23 8059 7673, Fax: (44)23 8059 3149, e-mail: kag@orc.soton.ac.uk
}

\begin{abstract}
Quadratic optical nonlinearities offer several attractive features for the implementation of ultra-fast, low-noise and transparent telecom devices. Technological progress has dramatically increased their efficiency, driving a steady move towards practical applications. In this talk we shall discuss the use of cascaded quadratic interactions in $\mathrm{LiNbO}_{3}$ integrated devices for pulse diagnostics, format conversion and signal regeneration in ultra-fast multi-wavelength telecom systems.
\end{abstract}

Keywords: All-optical switching, frequency-resolved optical gating, 3-R regeneration, nonlinear integrated optics, lithium niobate.

\section{INTRODUCTION}

Ultrafast all-optical applications, in which signal beams can be controlled by either their own intensity or by the temporal overlap with other beams, require nonlinear materials and interactions. In the early 1990s, seminal experiments on quadratic $\left[\chi^{(2)}\right]$ "cascading" pointed the way to a brand new range of viable devices for all-optical signal processing, mode-locking, pulse compression and solitons based on $\chi^{(2)}$ nonlinearities [1]. Since then, the maturity reached by the techniques for forming Quasi-Phase-Matching (QPM) gratings [2], and combining them with low-loss waveguides has increased the efficiency of quadratic interactions by orders of magnitude. This allows today's $\chi^{(2)}$ integrated devices [3] to rival even the record $\chi^{(3)}$ optical nonlinearities achieved through sophisticated fiber microstructuring and glass engineering [4].

In this paper we shall explore the potential of cascaded $\chi^{(2)}$ interactions for the implementation of novel alloptical devices operating with picosecond pulses in telecom systems. The devices we shall consider are based on the $\chi^{(2)}: \chi^{(2)}$ configuration of Fig. 1a, involving cascaded Second Harmonic Generation (SHG) and Difference Frequency Generation (DFG) occurring within the same medium. The scheme generates a new frequency $\left(\omega^{\prime}\right)$ from two input signals $\left(\omega\right.$ and $\left.\omega_{\mathrm{P}}\right)$. Overall, it mimics a $\chi^{(3)}$ four-wave mixing process: $\omega^{\prime}=\omega_{\mathrm{P}}+\omega_{\mathrm{P}}-\omega$ by means of two three-wave mixing steps, i.e. SHG: $\omega_{\mathrm{SH}}=\omega_{\mathrm{P}}+\omega_{\mathrm{P}}$, followed by DFG: $\omega^{\prime}=\omega_{\mathrm{SH}}-\omega$.

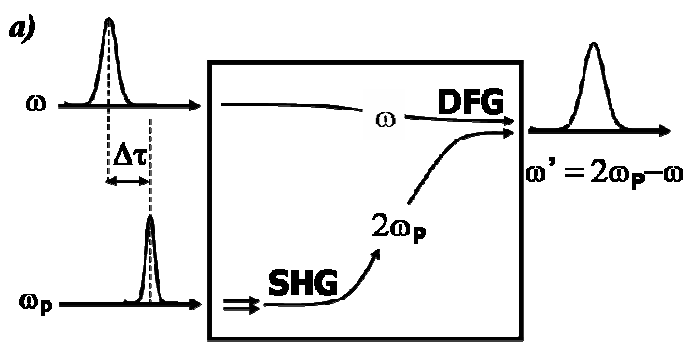

b)

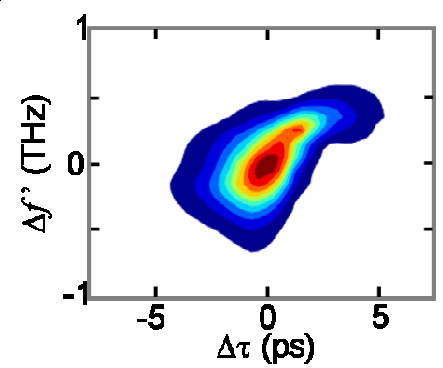

Figure 1. a) Sketch of the SHG:DFG scheme in the pulsed regime and b) spectrogram measured in FROG experiments (see fig. 2). $\Delta \tau=$ time delay between the input pulses, $\Delta f^{\prime}=$ frequency shift with respect to $f^{\prime}=2 f_{P^{-}}-$.

The SHG:DFG cascading scheme was first proposed for telecom applications and analysed in the continuouswave $(\mathrm{CW})$ and quasi-CW regime in Ref. [5]. Several experimental demonstrations and field applications have been achieved since [6].

Here we are considering devices which exploit the above configuration for signal processing of ultra-short pulses, in a regime where temporal walk-off effects are no longer negligible. We shall show how the cascading scheme can lead to novel devices for ultrafast all-optical processing in the third telecommunication window, overcoming the limitations imposed by temporal walk-off in standard single-step quadratic devices.

The numerical results we shall present are based on coupled mode equations [7], numerically solved via a Split Step Fourier algorithm [8]. The devices we shall discuss can in principle be implemented in any $\chi^{(2)}$ medium, but for a more quantitative evaluation of their performance we shall refer to a specific material

The work of. K. Gallo is supported by the Leverhulme Trust via an Early Career Fellowship (ECF/2004/0401). 
system, i.e. Periodically Poled $\mathrm{LiNbO}_{3}$ (PPLN) waveguides, which at the moment provide the most advanced technology platform for quadratic applications [3].

The following sections illustrate some possibilities offered by the configuration of Fig. $1 a$ for pulse characterisation (section 2), format conversion (section 3) and optical regeneration (section 4) in ultrafast telecom systems.

\section{FREQUENCY RESOLVED OPTICAL GATING (FROG)}

FROG is a well-established and widespread technique for the complete characterisation of ultra-short optical pulses [9]. Its standard implementation in quadratic media normally involves mixing two pulses in a frequency degenerate (SHG FROG) or non-degenerate (XFROG) configuration. The intensity and phase of the input pulses can be retrieved via suitable algorithms from measurements of the output spectra (around the frequency generated by the $\chi^{(2)}$ medium) as the time-delay $(\Delta \tau)$ between the input pulses is varied.

Single step $\chi^{(2)}$ FROG schemes (SHG, typically) suffer from a trade-off between time resolution and sensitivity imposed by the temporal walk-off of the interacting pulses within the device, due to their group velocity mismatch: the shorter the pulses $(\tau)$, the shorter the useful length of the device $(\mathrm{L})$. Unless special measures are taken to broaden the acceptance bandwidth [10], the maximum allowed device length for SHG FROG on a 1 ps pulse at $1.55 \mu \mathrm{m}$ in PPLN is limited to $\sim 3 \mathrm{~mm}$ (i.e. $\tau / \mathrm{L} \sim 0.3 \mathrm{ps} \cdot \mathrm{mm}^{-1}$ ). This considerably limits the conversion efficiency, hence the FROG sensitivity. Guided-wave configurations can increase the sensitivity [11], but do not overcome the intrinsic limitations imposed by walk-off. A recent analysis of cascaded SHG:DFG in the ultrashort pulse regime highlighted the possibility to ease the above constraints and actually use devices of several walk-off lengths for high resolution FROG on picosecond and even shorter pulses [3]. These predictions were confirmed by experiments at telecommunications wavelengths in a PPLN waveguide [12], whose fabrication conditions are detailed in Ref. [11].

The FROG experiments were carried out with the setup sketched in Fig. $2 a$. A mode-locked fiber laser operating at $1541.9 \mathrm{~nm}$ generated a train of picosecond pulses at $2.5 \mathrm{GHz}$ from which both inputs to the FROG device (pump and test signals) were produced. The path producing the pump pulses for SHG $\left(\lambda_{\mathrm{P}}=1541.9 \mathrm{~nm}\right)$ included a motorised delay line $(\Delta \tau)$, an amplifier (EDFA) and a filter (BPF) to suppress the ASE. The test pulses were obtained in a separate path by wavelength-converting the original signal to $\lambda_{\omega}=1560 \mathrm{~nm}$ and then amplifying them. The average powers for the pump and test signals, recombined at the input of the $\chi^{(2)}: \chi^{(2)}$ FROG device, were $100 \mathrm{~mW}$ and $0.2 \mathrm{~mW}$, respectively. The FROG spectrograms were measured around $\lambda_{\omega}{ }^{\prime}=$ $1524.2 \mathrm{~nm}($ Fig. $1 b)$. The intensity and chirp of the test pulses, as retrieved via a blind-deconvolution algorithm [13], are shown in Fig. 2b. Independent measurements in the time and frequency domain confirmed the highquality of the FROG retrieval for $2.1 \mathrm{ps}$ test pulses (with energies as low as $80 \mathrm{fJ}$ ). By contrast, a standard SHG FROG scheme in the same waveguide would have been limited to minimum pulse-durations of $\sim 8 \mathrm{ps}$.
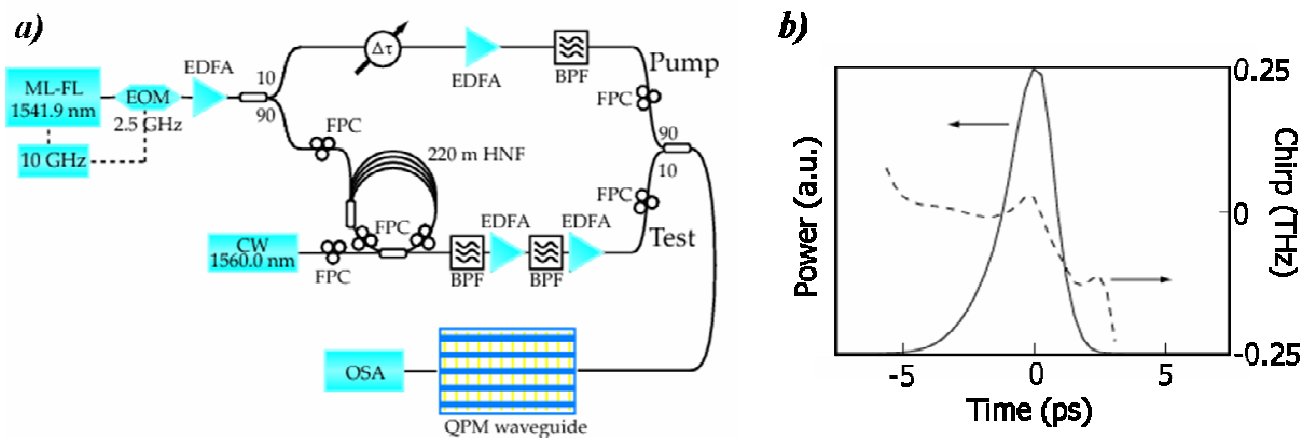

Figure 2. a) Experimental setup used for the FROG measurements at $2.5 \mathrm{GHz}$, with test $\left(\lambda_{\omega}=1560 \mathrm{~nm}\right)$ and pump $\left(\lambda_{P}=1541.9 \mathrm{~nm}\right)$ average pulse powers of $0.2 \mathrm{~mW}$ and $100 \mathrm{~mW}$, respectively. $\left.b\right)$ Retrieved test pulse.

\section{TDM TO WDM FORMAT CONVERSION}

Efficient ways to convert telecommunications signals between time and frequency domains would make it possible to develop systems in which optical processing steps could be performed in whichever domain provides the best performance and/or the most practical implementation. For instance, an efficient TDM to WDM format converter could translate such a fundamental function as add-drop multiplexing from the time domain (where it requires critical synchronization and state-of-the-art ultrafast switches) to the wavelength domain (where it can be readily performed using commercially available passive optical filters). 
Recently, TDM $\rightarrow$ WDM format conversion based on $\chi^{(3)}$ cross-phase modulation in nonlinear fibers has been demonstrated [14]. Here we investigate an alternative solution, based on the $\chi^{(2)}: \chi^{(2)}$ configuration sketched in Fig. $3 a$, in which cascaded SHG and DFG are used to map the individual tributary channels from an incoming TDM stream at $\omega_{\mathrm{P}}\left(\right.$ timeslots $\mathrm{t}_{1 \cdot \cdot \mathrm{N}}$, with $\mathrm{N}=4$ in Fig. $3 a$ ) onto $\mathrm{N}$ separate wavelengths $\left(\omega_{1} \cdot \mathrm{N}^{\prime}\right)$ to produce at the output a mixed TDM-WDM replica with the same line rate of the TDM input $\left(\mathrm{T}_{\mathrm{REP}}\right)$. The format conversion is accomplished by means of SHG from the TDM stream, followed by DFG between the SH of the TDM signal and a linearly chirped input pulse (covering $\mathrm{N}$ time slots of the TDM input stream) simultaneously sent into the device. Each of the SH TDM pulses interacts with a different portion of the input chirped pulse and sees a different instantaneous frequency $\left(\omega_{1 \cdot{ }_{\mathrm{N}}}\right)$, from which the $\mathrm{N}$ output WDM channels $\left(\omega_{1 \cdot \mathrm{N}^{\prime}}=2 \omega_{\mathrm{P}}-\omega_{1 \cdot . \mathrm{N}}\right)$ are generated. Numerical simulations for a TDM $\rightarrow$ WDM converter in a 3-cm long PPLN channel waveguide with an SHG efficiency of $80 \% \mathrm{~W}^{-1} \mathrm{~cm}^{-2}$ are shown in Fig. $3 a-b$. The device inputs (Fig. $3 a$ ) are: a $40 \mathrm{~Gb} / \mathrm{s}$ TDM signal (four $10 \mathrm{~Gb} / \mathrm{s}$ tributary channels) at $\lambda_{\omega}=1546 \mathrm{~nm}$, consisting of 5 ps Gaussian pulses with a peak power of $150 \mathrm{~mW}$, and a $10 \mathrm{GHz}$ train of linearly chirped $(0.06 \mathrm{~nm} / \mathrm{ps})$ rectangular pulses with a time duration of $\sim 100 \mathrm{ps}$ and a peak power of $30 \mathrm{~mW}$, around $\lambda_{\omega}=1551 \mathrm{~nm}$. Fig. $3 b$ shows the calculated mixed TDM-WDM output, which maps the input channels onto 4 separate WDM carriers $\left(\lambda_{\omega}{ }^{\prime}=\right.$ 1538.9, 1540.4, 1541.9 and $1543.4 \mathrm{~nm}$ ), while retaining the $40 \mathrm{~Gb} / \mathrm{s}$ line rate of the TDM input. The simulations predict $5.6 \mathrm{ps}$ output pulses with peak powers of $\sim 3 \mathrm{~mW}$.
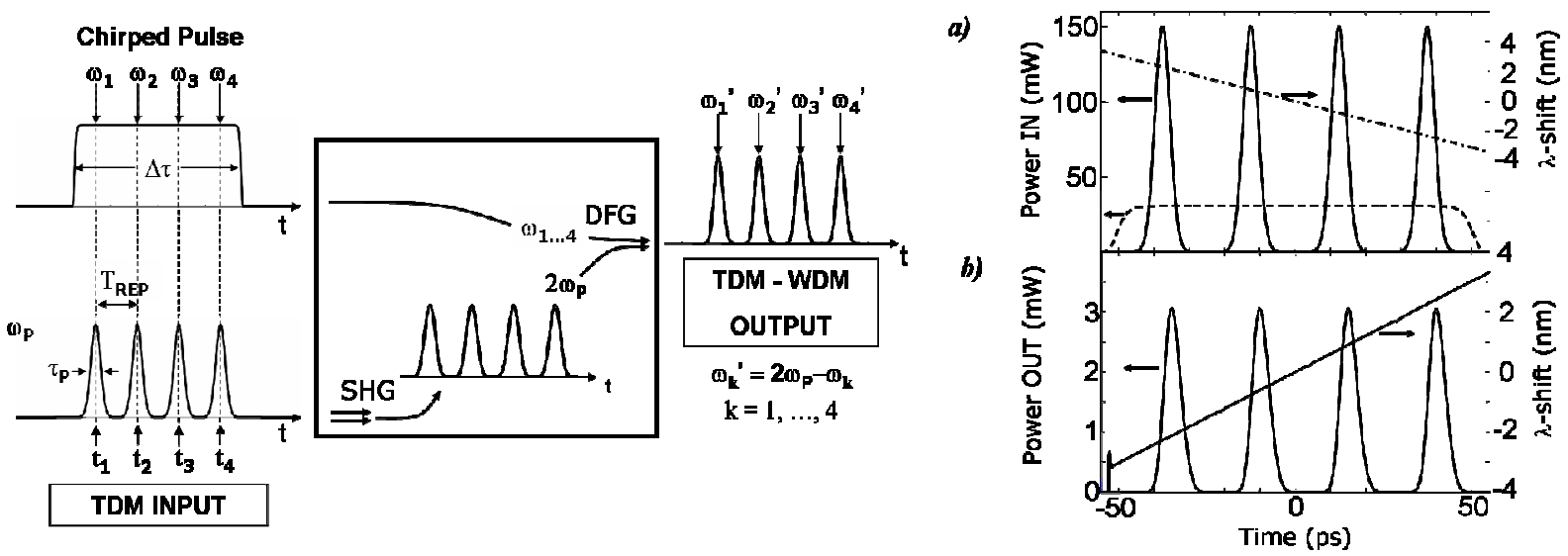

Figure 3. Sketch of the structure of the SHG:DFG TDM to WDM converter. a) Input and b) output signals, calculated for a 3-cm long device in a PPLN waveguide with a SHG efficiency of $80 \% \mathrm{~W}^{1} \mathrm{~cm}^{-2}$.

\section{SIGNAL RETIMING AND RESHAPING}

For advanced transmission systems, 3-R regeneration (re-amplification, re-shaping and re-timing) is needed to combat the transmission impairments associated with waveform distortions due to fiber transmission as well as with noise and jitter accumulation. Amplification can be readily performed in-line in fiber systems, but reshaping and re-timing, especially for high bit rate signals, will require novel approaches. Most of the all-optical retiming and reshaping techniques explored so far for ultrafast networks are based on third-order nonlinearities. One particular implementation [15-16], involves broadening the incoming noisy signal to create longer flat-top pulses which are then gated in a nonlinear switch by a clock signal recovered from the incoming data stream. Provided the input signal is transformed into a low-ripple rectangular pulse with the proper timeduration, the timing and the shape of the clock are then faithfully transferred onto the output data stream.

The use of the SHG:DFG scheme for picosecond pulse retiming has been recently considered in ref. [17]. In principle the SHG:DFG device could perform both the preliminary rectangular pulse shaping (via SHG) and the subsequent nonlinear switching (via DFG). In fact, SHG in a $\chi^{(2)}$ medium whose length exceeds the walkoff limit would produce a broadened SH replica of the incoming noisy data stream (at $\left.\omega_{\mathrm{P}}\right)$, while DFG between the broadened SH and an incoming clock signal (at $\omega$ ) would restore, via parametric transfer from the clock, the correct timing and shape on the output signal $\left(\right.$ at $\left.\omega^{\prime}=2 \omega_{\mathrm{P}}-\omega\right)$. Unfortunately, numerical analyses show that it is practically impossible to optimize the device for good timing jitter suppression and efficient conversion, when attempting to achieve both pulse shaping and switching via the SHG:DFG cascaded interactions [17].

On the other hand, the two steps (rectangular pulse shaping and switching) can be conveniently dissociated and independently optimized, e.g. by using superstructured Bragg fiber gratings for pulse shaping and optical 
nonlinearities for switching, as done in Ref. [16]. This alternative approach, applied to the $\chi^{(2)}: \chi^{(2)}$ case, is illustrated in Fig. 4.

In Fig. 4, the noisy input train of pulses at $\omega_{\mathrm{P}}$ are preliminarily reshaped into longer rectangular pulses (pulsewidth: $\tau_{\mathrm{P}}$, timing jitter: $\delta t_{\mathrm{P}}$ ), which then pump the SHG process in the $\chi^{(2)}: \chi^{(2)}$ device. The SH data stream (still retaining the timing jitter and noise of the input) is then gated by the input clock (pulsewidth: $\tau$, no timing jitter) via DFG. Fig. $3 a$ and $b$ show the calculated peak power ( $\left.\mathrm{P}^{\prime}\right)$ and temporal position of the output pulse ( $\left.\mathrm{t}^{\prime}\right)$ as a function of the input jitter $\left(\delta \mathrm{t}_{\mathrm{P}}\right)$, for $160 \mathrm{~Gb} / \mathrm{s}$ operation $\left(\mathrm{T}_{\mathrm{REP}}=6.3 \mathrm{ps}\right)$. The solid lines correspond to the case in which the input TDM pulses have been preliminarily reshaped into rectangular pulses ( $\tau_{\mathrm{P}}=3 \mathrm{ps}$ ), while the dashed lines correspond to the case where the SHG:DFG device is directly driven by the input signal (1.5 ps FWHM Gaussian pulses) as in Ref. [17]. For both cases we assumed the same average power for the input at $\omega_{\mathrm{P}}$, corresponding to peak SHG pump powers of $500 \mathrm{~mW}$ and $1 \mathrm{~W}$ for the $3 \mathrm{ps}$ and $1.5 \mathrm{ps}$ pulses, respectively. The clock signal was a regular train of Gaussian pulses with a FWHM $\tau=1.5 \mathrm{ps}(100 \mathrm{~mW}$ peak power) at 160 GHz. The parameters of the $\chi^{(2)}$ medium used in the simulations correspond to a $2 \mathrm{~cm}$-long PPLN waveguide with an $80 \% \mathrm{~W}^{-1} \mathrm{~cm}^{-2} \mathrm{SHG}$ efficiency. The zero on the $\delta \mathrm{t}_{\mathrm{p}}$ axis has been chosen to coincide with the point where the most efficient conversion to $\omega^{\prime}$ occurs (i.e. maximum of $\mathrm{P}^{\prime}$ ).

The curves in Fig. $4 a-b$ show a clear improvement of the retiming characteristics of the device (i.e. a much flatter $t$ '-response) associated with the preliminary reshaping of the input signal pulses.
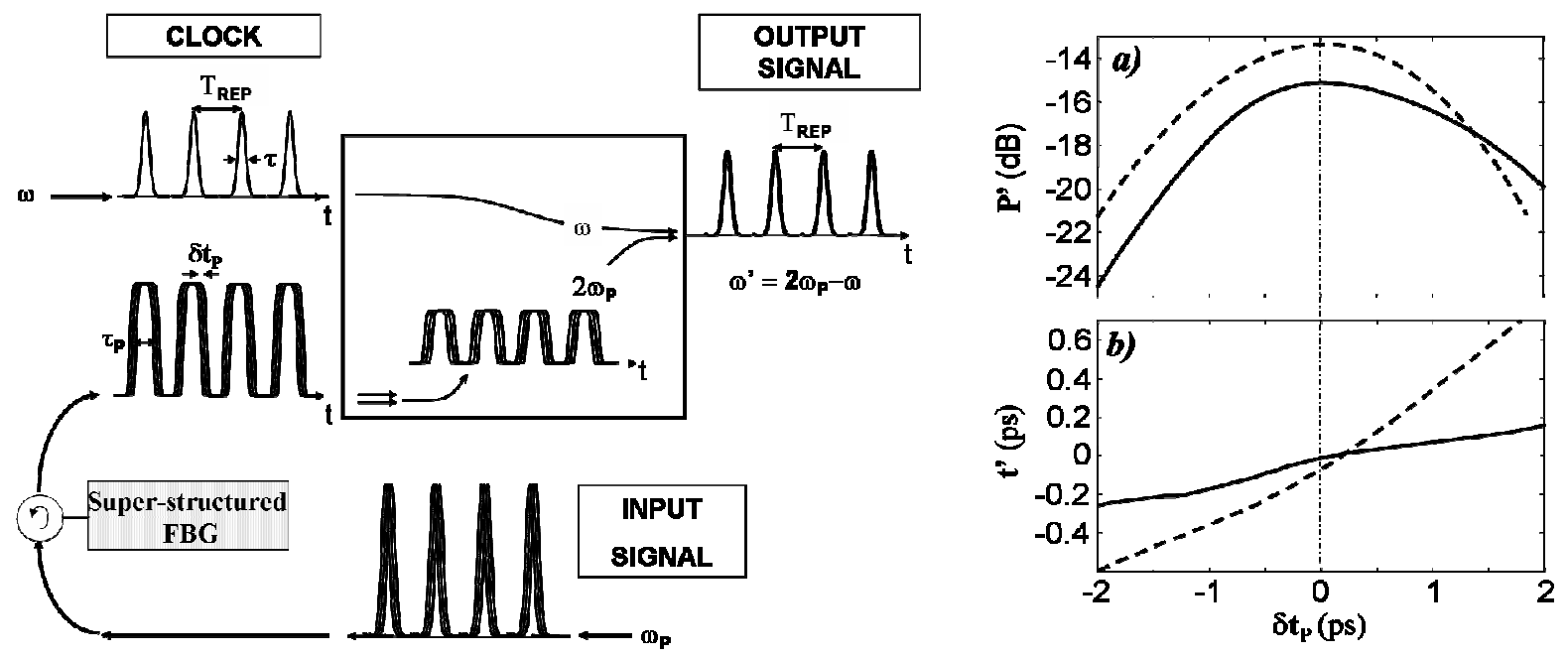

Figure 4. Sketch of the retiming and reshaping $\chi^{(2)}: \chi^{(2)}$ device. a) Peak power, $P^{\prime}$, and b) temporal position, $t^{\prime}$, of the output pulse as a function of the input jitter $\left(\delta t_{P}\right)$ with (-) and without (--) pre-shaping of the input signal.

\section{CONCLUSIONS AND FUTURE WORK}

We have proposed and investigated the performance of several devices for all-optical signal processing in ultrafast photonic networks via cascaded $\chi^{(2)}$ nonlinearities. The theoretical predictions for a SHG:DFG FROG device have been confirmed by experiments carried out on picosecond pulses at telecom wavelengths in a PPLN channel waveguide. The same technology platform could also allow the demonstration of the novel $\chi^{(2)}: \chi^{(2)}$ devices for TDM to WDM conversion and 3-R signal regeneration presented in this paper.

\section{REFERENCES}

[1] G. I. Stegeman and W. E. Torruellas, "Nonlinear materials for information processing and telecommunications", Phil. Trans. R. Soc. London A 354, 745 (1996).

[2] J. A. Armstrong, N. Bloembergen, J. Ducuing, and P. S. Pershan, "Interactions of between light waves in a nonlinear dielectric", Phys. Rev. 127, 1918 (1962).

[3] K. R. Parameswaran, R. K. Route, J. R. Kurz, R. V. Roussev, M. M. Fejer, and M. Fujimura, "Highly efficient second-harmonic generation in buried waveguides formed by annealed and reverse proton exchange in periodically poled lithium niobate", Opt. Lett. 27, 179 (2002).

[4] J.Y.Y. Leong et al., OFC 2005 Anaheim 6-11 Mar 2005, paper PDP22.

[5] K. Gallo, G. Assanto and G.I. Stegeman, "Efficient wavelength shifting over the erbium amplifier bandwidth via cascaded second order processes in lithium niobate waveguides", Appl. Phys. Lett. 71, 
1020 (1997); K. Gallo and G. Assanto, "Analysis of lithium niobate all-optical wavelength shifters for the third spectral window”, J. Opt. Soc. Am. B, 16, 741 (1999).

[6] See for instance: M. H. Chou, I. Brener, M. M. Fejer, E. E. Chaban, and S. B. Christman, "1.5- $\mu$ m-band wavelength conversion based on cascaded second-order nonlinearity in $\mathrm{LiNbO}_{3}$ waveguides", IEEE Photon. Technol. Lett. 11, 653 (1999); Y. L. Lee, H. Suche, J. H. Lee, W. Grundkotter, V. Quiring, and W. Sohler, "Wavelength- and time-selective all-optical channel dropping in periodically poled Ti: $\mathrm{LiNbO}_{3}$ channel waveguides", IEEE Photon. Technol. Lett. 15, 978 (2003).

[7] J. Prawiharjo, K. Gallo, N. G. R. Broderick, and D. J. Richardson, "Frequency resolved optical gating in the $1.55 \mu \mathrm{m}$ band via cascaded $\chi^{(2)}$ processes", J. Opt. Soc. Am. B 22, 1985 (2005).

[8] G. P. Agrawal, Nonlinear Fiber Optics, $3^{\text {rd }}$ ed., Academic Press, 2001.

[9] R. Trebino, "Frequency Resolved Optical Gating: the measurement of ultrashort laser pulses", Kluwer Academ. Publ., London, 2002.

[10] S.-D. Yang, A. M. Weiner, K. R. Parameswaran, and M. M. Fejer, "Ultrasensitive second-harmonic generation frequency-resolved optical gating by aperiodically poled $\mathrm{LiNbO}_{3}$ waveguides at $1.5 \mu \mathrm{m}$ ", Opt. Lett. 30, 2164 (2005).

[11] J. Prawiharjo, K. Gallo, B. C. Thomsen, M. A. F. Roelens, P. J. Almeida, N. G. R. Broderick, and D. J. Richardson, "Frequency resolved optical gating in a quasi-phase-matched $\mathrm{LiNbO}_{3}$ waveguide", Photon. Technol. Lett. 17, 849 (2005).

[12] J. Prawiharjo, F. Parmigiani, K. Gallo, P. Petropoulos, N. G. R. Broderick, and D. J. Richardson, "Cascaded- $\chi{ }^{(2)}$-interaction-based frequency-resolved optical gating in a periodically poled $\mathrm{LiNbO}_{3}$ waveguide", Opt. Lett. 31, 244 (2006).

[13] D. J. Kane, G. Rodriguez, A. J. Taylor, and T.S. Clement, "Simultaneous measurement of two ultrashort laser pulses from a single spectrogram in a single shot", J. Opt. Soc. Am. B 14, 935 (1997).

[14] P. J. Almeida, P. Petropoulos, B. C. Thomsen, M. Ibsen, and D. J. Richardson, "All-optical packet compression based on time-to-wavelength conversion", IEEE Photon. Technol. Lett. 16, 1688 (2004).

[15] S. Watanabe, F. Futami, R. Okabe, Y. Takita, S. Ferber, R. Ludwig, C. Schubert, C. Schmidt, and H. G. Weber, "160 Gbit/s optical 3R-regenerator in a fiber transmission experiment", in Proc. OFC 2003, paper PD16-1-3 (2003).

[16] F. Parmigiani, P. Petropoulos, M. Ibsen, and D. J. Richardson, "All-optical pulse reshaping and retiming systems incorporating pulse shaping fiber Bragg grating”, J. Lightwave Technol. 24, 357 (2006).

[17] T. Hasegawa, X. Wang, and A. Suzuki, "Retiming of picosecond pulses by a cascaded second-order nonlinear process in quasi-phase-matched $\mathrm{LiNbO}_{3}$ waveguides", Opt. Lett. 29, 2776 (2004). 